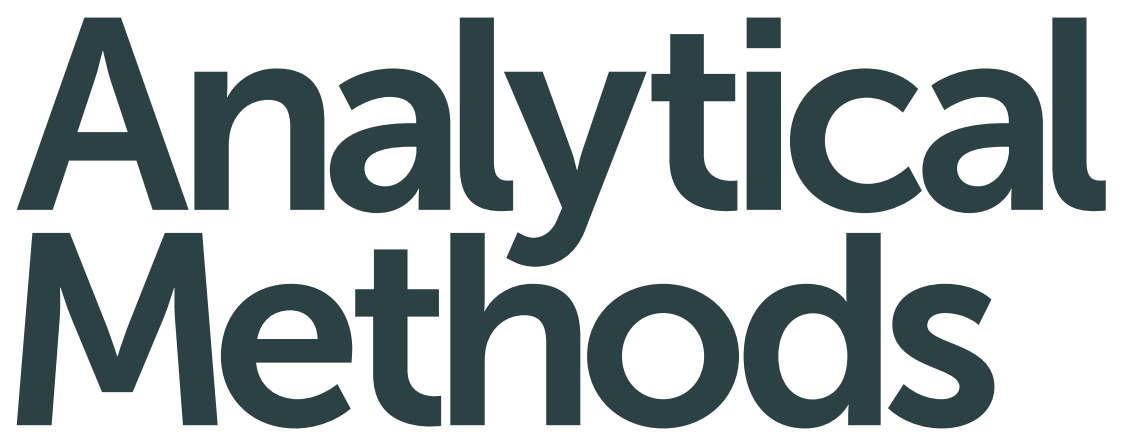

www.rsc.org/methods



ISSN 1759-9660

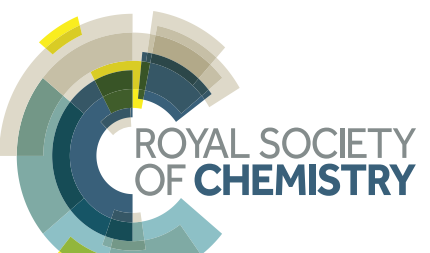




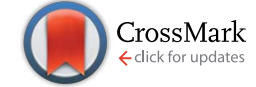

Cite this: Anal. Methods, 2015, 7, 3664

\author{
Vjekoslav Kokoric, Andreas Wilk and Boris Mizaikoff*
}

\section{iPRECON: an integrated preconcentrator for the enrichment of volatile organics in exhaled breath $\uparrow$}

Received 11th February 2015

Accepted 19th March 2015

DOI: $10.1039 / c 5 a y 00399 g$

www.rsc.org/methods

In this study, a new generation of integrated preconcentrators particularly suited for exhaled breath gas analysis is described. The developed analyzer system comprises a compact preconcentrator (iPRECON) exemplarily tested for sampling isoprene, which readily couples to substrate-integrated hollow waveguides (iHWGs) of the same footprint serving simultaneously as highly miniaturized gas cell and photon conduit in combination with a compact infrared spectrometer.

Exhaled breath gas analysis is a strategy of increasing importance in medical diagnostics for early disease detection and therapy progress monitoring. ${ }^{1}$ Because of its non-invasiveness and ease of sampling, new sensing methods continuously emerge. Evidently, the rather minute concentrations (i.e., low ppm to ppt levels) of volatile organic constituents (VOCs) appearing in the exhaled breath matrix pose a challenge to many sensing techniques, and in particular to mid-infrared (MIR; 2-20 $\mu \mathrm{m}$ ) absorption spectroscopic techniques. This is especially true whenever an application requires the usage of a rather compact (e.g., hand-held) sensor, thereby limiting the useful absorption path length. ${ }^{2}$

Here, we take advantage of preconcentration as one possible solution to enhance the analytical signal without extending the absorption path length..$^{3-5}$ Specifically, isoprene - a widely recognized marker for cholesterol metabolism disorders ${ }^{6-10}$ was previously detected via substrate-integrated hollow waveguide (iHWG) based IR-spectroscopy after enrichment within an appropriate adsorbent material, and subsequent thermal desorption into the detection system. iHWGs are a new generation of hollow waveguides, which are suitable for simultaneous use as a waveguide, and as a highly miniaturized gas cell. ${ }^{11,12}$ Hence, iHWGs are highly attractive alternatives for

Institute of Analytical and Bioanalytical Chemistry, University of Ulm, Albert-Einstein-Allee 11, 89081 Ulm, Germany; Web: http://www.uni-ulm.de/iabc. E-mail: boris.mizaikoff@uni-ulm.de; Fax: +49-731-50-22763; Tel: +49-731-5022750 $\dagger$ Electronic supplementary information (ESI) available. See DOI: 10.1039/c5ay00399g conventional multi-pass gas cells in spectroscopic gas sensing applications that require rapid sample transient times, minute sample volumes, and a small device footprint. ${ }^{13-15}$

The concentration of isoprene in exhaled human breath is usually too low for direct detection via MIR absorption spectroscopy. According to Fenske et al., ${ }^{16}$ the highest concentrations for endogenous constituents in exhaled breath are reported for isoprene (12-580 ppb), ethanol (13-1000 ppb), methanol (160-2000 ppb), and acetone (1.2-1800 ppb); other alcohols just appear at very low parts per billion (ppb) concentrations. Therefore, a reproducible enrichment step prior to the analysis is frequently required, and in particular for selectively enhancing the isoprene signal. ${ }^{17,18}$ During preliminary studies testing various preconcentration concepts, our research group has readily demonstrated the potential determination of isoprene in human breath using a cylindrical tube packed with an adsorbent serving as preconcentrator. Thus obtained results revealed that the detection of isoprene by mid-infrared spectroscopy is indeed feasible, if an appropriate preconcentration step is included. ${ }^{5}$ However, this setup required elaborate transfer of the desorbed sample into the iHWG, was neither compact nor integrated or automated, and did not allow for an integrated heating solution (i.e., using heating tape wrapped around the cylindrical preconcentrator). Consequently, nextgeneration preconcentrator devices should provide improved temperature control during the desorption process, automated valve operation, and optimized cooling. In addition, the device should ideally match the footprint of the iHWG for future integration of active transducer and preconcentrator. Hence, a channel structure containing the sorbent material was integrated into a substrate with dimensions of $75 \times 50 \mathrm{~mm}$ (i.e., similar to the iHWG), and closed off with a lid plate. Last but not least, this design enables rapid and facile packing/exchange of any kind of sorbent material within the channel.

In the present study, isoprene was selected as an exemplary yet relevant volatile organic constituent, which is frequently determined in exhaled human breath. Given the flexibility of the device, different adsorbent materials suitable for the 
enrichment of other biomarkers ${ }^{16,19,20}$ in human breath may readily pack into the structure.

Fig. 1 shows a 3D rendered model of the main components of the preconcentrator. The device is milled from a $60 \times 90 \times$ $10 \mathrm{~mm}$ aluminum block. Aluminum provides excellent thermal conductivity of approximately $237 \mathrm{~W} \mathrm{~m}^{-1} \mathrm{~K}^{-1}$, a low density of $2.7 \mathrm{~g} \mathrm{~cm}^{-3}$, and the ability to resist corrosion, which are essential properties for a preconcentrator. Besides the low density, which facilitates a lightweight construction, aluminum also resists mechanical stress. Furthermore, iHWG structures are currently predominantly fabricated from aluminium, thus providing matching materials for future stacking/integration of the devices.

The arrows in Fig. 1 indicate the sections where the material was actually processed. (A) provides a groove structure for housing an O-ring (50 $\mathrm{mm}$ diameter), thereby sealing the preconcentrator against the bottom plate. (B) shows the channel structure that may be packed with any kind of sorbent material (here, Dowex Optipore L493, Sigma-Aldrich Chemie GmbH, Taufkirchen b. M., Germany). (C) are threaded holes for mounting the bottom plate. (D) indicates the cooling structure facilitating heat dissipation, currently manufactured with a width of $3 \mathrm{~mm}$ and a depth of $1 \mathrm{~mm}$ per fin. Two commercially available CPU fans were mounted on the top of the device enabling rapid and controlled cooling of the preconcentrator. Via the threaded holes (E), Luer-Lock connectors were mounted on both sides to connect the preconcentrator with computercontrolled three-way-valves (T161PK031, NResearch Inc., West Caldwell, USA) (Fig. 2 (E)).

The entire experimental setup - iPRECON plus iHWG coupled to a FT-IR spectrometer - is schematically shown in Fig. 3 comprising (A) a Bruker IRcube FT-IR spectrometer (Bruker Optics Inc., Ettlingen, Germany), (B) a liquid nitrogen cooled mercury-cadmium-telluride (MCT) detector (Infrared Associates, USA), (C and D) an iHWG as previously developed by our research team, ${ }^{3}$ (E and F) gold-coated mirrors (Thorlabs, USA), (G) a gas mixing system, and (H) the iPRECON. The green line indicates the direction of the gas flow initiated by the gas mixing system (GMS; developed in collaboration with LLNL-Lawrence Livermore National Laboratory, Livermore, USA), i.e., the path of the carrier gas $\left(\mathrm{N}_{2}\right)$ and sample during a measurement.

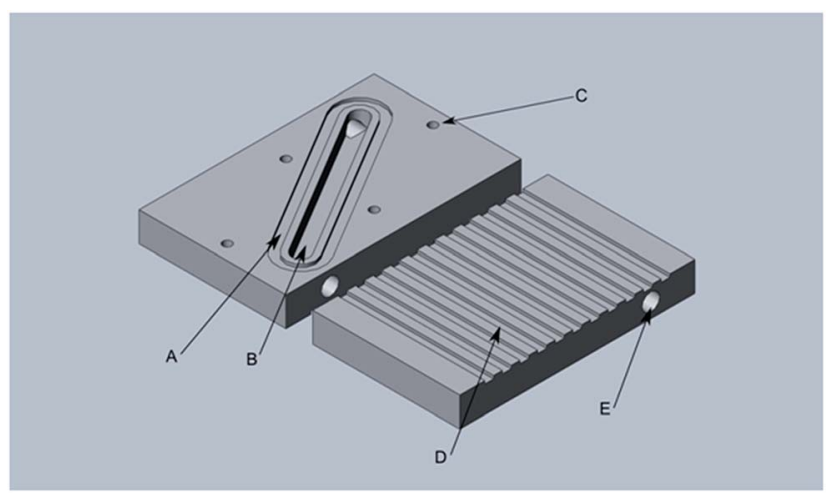

Fig. 1 Top part of the preconcentrator: (A) O-ring channel, (B) sorbent channel, (C) mount threads, (D) cooling structures, (E) Luer-Lock connector threads.

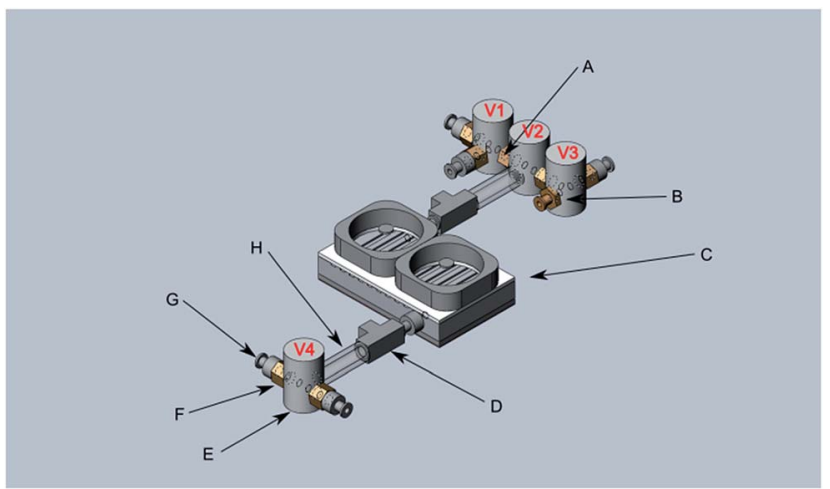

Fig. 2 Functional iPRECON overview; (A) direct valve connectors, (B) Luer-Lock connector, (C) preconcentrator, (D) three-way-valve, (E) solenoid valves (V1-V4), (F) imperial to metric adapter, (G) M5 to LuerLock adapter, $(\mathrm{H})$ tubing

Different possible pathways of the gas flow for e.g., measuring, sampling, purging, etc. are shown in the ESI. $\uparrow$ Red lines illustrate the optical path, i.e., propagation of the IR radiation.

Emitted IR radiation from the FT-IR spectrometer (A) was first reflected by a planar gold-coated mirror (E) onto a goldcoated off-axis parabolic mirror (OAPM) (F), and then focused into the iHWG (D). The radiation propagated through the iHWG (D), which simultaneously served as a miniaturized gas cell and as a hollow waveguide. IR radiation emanating at the distal end of the iHWG was then focused onto a MCT detector (B). Via the gas inlet and outlet ports of the iHWG, the optical system was connected to the iPRECON system (H).

A certified gas standard of isoprene (nominal concentration: $100 \mathrm{ppm}$ in $\mathrm{N}_{2}$; vendor: Westfalen AG, Weißenhorn, Germany) was diluted with nitrogen to a minimum concentration of $1 \mathrm{ppm}$ using a custom-made mass flow-controlled gas mixing unit prototype developed in collaboration with Lawrence Livermore National Laboratory (LLNL; Livermore/CA, USA), and fed into the iPRECON.

Fig. 4 provides an overview on the data generation and data processing routines for evaluating isoprene concentrations in

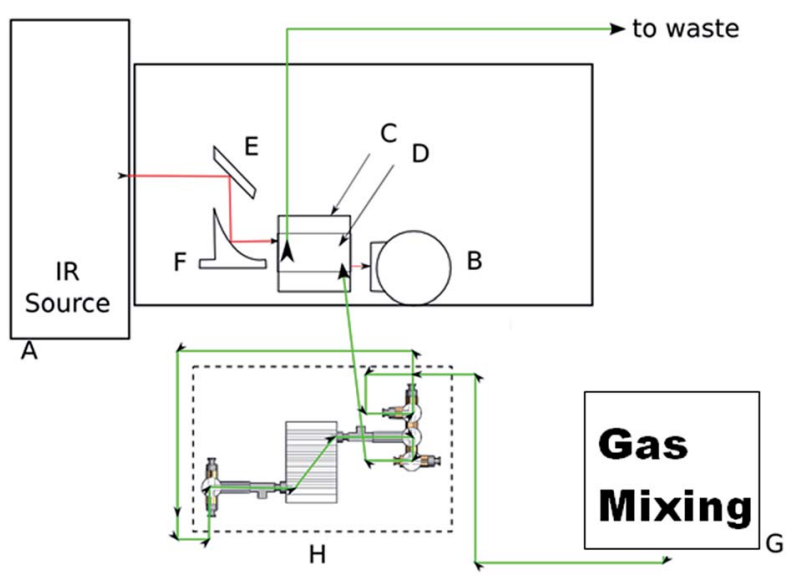

Fig. 3 Experimental setup: (A) IR source (here, FT-IR spectrometer), (B) MCT detector, (C) iHWG mount, (D) iHWG, (E) gold-coated planar mirror, (F) gold-coated off-axis parabolic mirror, (G) gas mixing system (GMS), (H) iPRECON. 

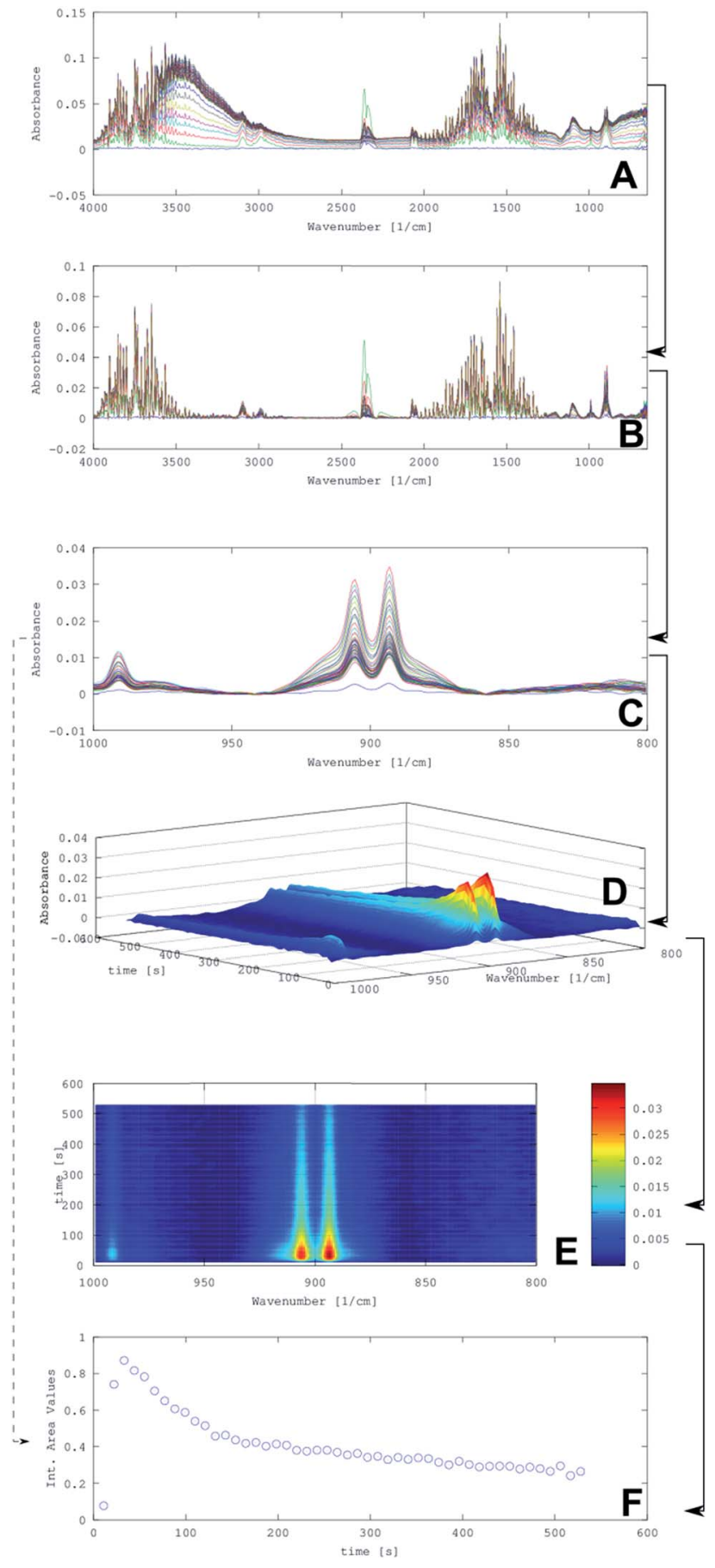

Fig. 4 Flow chart of data generation and data processing using the isoprene gas standard diluted to $10 \mathrm{ppm}$ : (A) raw IR spectra, (B) baseline corrected spectra, (C) reduction to the relevant spectral region (i.e., isoprene signature), (D) 3D plot of the spectra as isoprene is desorbed from the iPRECON into the iHWG, (E) contour plot derived from data in (D), (F) integrated values of the isoprene absorption peak $\left(x-y \mathrm{~cm}^{-1}\right)$ peak vs. time.

exhaled breath extracted from the obtained IR spectra after preconcentration and desorption. Using an example (i.e., isoprene concentration of $10 \mathrm{ppm}$ ), the data processing routine from the obtained raw IR spectra (A) to the integrated peak values (F) are illustrated.
In (A), the raw data of 50 individual spectra collected from one isoprene sample are shown in a stacked representation. Evidently, a baseline drift is noticeable, especially in the spectral region around $3500 \mathrm{~cm}^{-1}$, which results from water absorptions. Hence, in a first step the baseline was corrected resulting in the processed spectra shown in (B). The correction was performed via a Matlab script developed for this purpose. This algorithm estimates the baseline of the raw spectrum via asymmetric least squares smoothing of the raw and a virtual baseline. $^{21}$ After subtraction of the virtual (i.e., calculated) baseline from the raw spectrum, the corrected spectrum is achieved. In a next step, the spectral region of interest was selected from (B); here, the isoprene absorption region from 800-1000 $\mathrm{cm}^{-1}$ corresponding to the bending vibrations of isoprene with maxima at 895 and $905 \mathrm{~cm}^{-122,23}$ to confine the spectral region of interest (C). Next, information on the isoprene concentration was extracted from each spectrum using a previously established isoprene calibration function (C).

Based on the relevant spectral region shown in (C), information on the concentration, i.e., the integrated isoprene peak values using 870 and $940 \mathrm{~cm}^{-1}$ as integration boundaries were obtained (illustrated by the dotted line from (C-F)). Hence, every point in $(\mathrm{F})$ is a result of the integration of one spectrum in (C). After this operation, a time-dependent concentration profile of the isoprene desorption into the iHWG may be extracted.

Alternatively, the dependence of the isoprene concentration on the desorption kinetics were obtained by visualizing the evolvement of the isoprene IR signature as a 3D plot (D). The 3D plot (D), which was produced by using the software package Octave, illustrates the change of absorption $v s$. time. Alternatively, absorption $v s$. time may be represented as a contour plot (E) providing the same information.

Fig. 5 illustrates the comparison of preconcentrated isoprene sample with a concentration of $1 \mathrm{ppm} v s$. a sample containing $100 \mathrm{ppm}$ of isoprene that was not preconcentrated, i.e., directly analysed using the iHWG in the spectral region from $800-1000 \mathrm{~cm}^{-1}$. The results for isoprene quantification using the iPRECON are summarized in Table 1 including the limit of

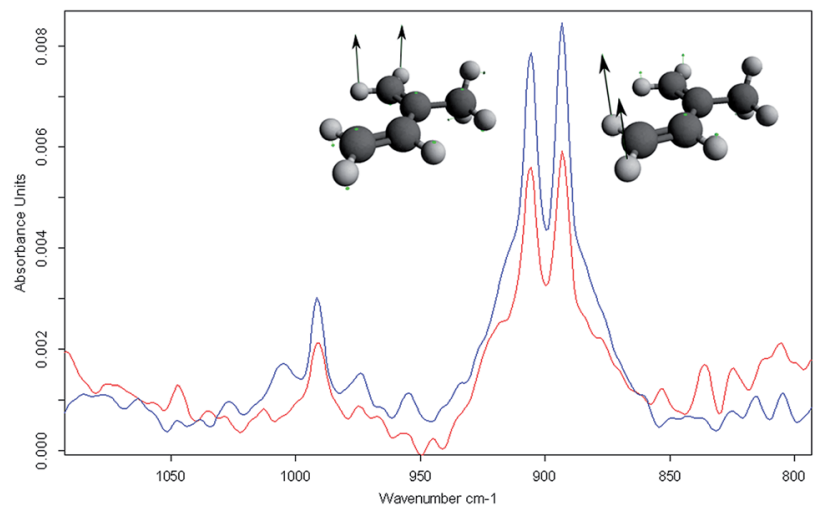

Fig. 5 Comparison of an averaged preconcentrated $1 \mathrm{ppm}$ isoprene sample (red), and a directly analysed (i.e., without preconcentration) $100 \mathrm{ppm}$ isoprene sample (blue). The isoprene molecules on the left and right side of the absorption peaks illustrate the associated bending vibrations at 895 and $905 \mathrm{~cm}^{-1}$, respectively. 
Table 1 Summarized analytical parameters of iPRECON

\begin{tabular}{ll}
\hline Analytical parameters & iPRECON \\
\hline Slope of the calibration & $0.083 \mathrm{~cm}^{-1} \mathrm{ppm}^{-1}$ \\
$R^{2}$ & 0.999 \\
LOD & $260 \mathrm{ppb}$ \\
LOQ & $867 \mathrm{ppb}$ \\
Preconcentration factor (PCF) & $>50$ \\
Heating time (s) & 250 \\
Cooling time (s) & 500
\end{tabular}

detection (LOD), the limit of quantification (LOQ) and the achievable preconcentration factor (PCF). To estimate the LOD/ LOQ, samples without analyte were measured to calculate the standard deviation of the blanks. According to Long et al. ${ }^{24}$, the LOD is defined as three times the standard deviation of the blank and the LOQ as ten times the standard deviation, respectively. All obtained figures-of-merit compare favourably to previously obtained results. $^{7}$

Using the iPRECON, a LOD for isoprene of $260 \mathrm{ppb}$ and a LOQ of $867 \mathrm{ppb}$ was determined. Compared to the system without preconcentration, a preconcentration factor of at least 50 was derived.

\section{Conclusions}

In the present study, we demonstrated a new generation of miniaturized preconcentrators that are suitable for enriching volatile organic constituents relevant e.g., in exhaled breath gas analysis. While the obtained results for the exemplary analyte isoprene are comparable to previously published results using conventional preconcentration strategies, the iPRECON offers several distinct advantages in terms of automation, user friendliness, reproducibility, compactness, and robustness. In addition, the iPRECON readily combines with the iHWG previously developed by our research team, thus offering an integrated solution for advanced mid-infrared gas sensing devices applicable not only in biodiagnostics, but also in environmental monitoring, atmospheric studies, security/surveillance applications, and process analysis.

\section{Acknowledgements}

The Machine Shop at University of Ulm is thanked for support during prototype development of iHWG and iPRECON. This work was performed in part under the auspices of the U.S. Department of Energy by Lawrence Livermore National Laboratory (LLNL) under Contract DE-AC52-07NA27344. This project was funded under LLNL sub-contract nos B603018 and B607114.

\section{Notes and references}

1 F. M. Musteata, TrAC, Trends Anal. Chem., 2013, 45, 154-168. 2 P. Jouy, M. Mangold, B. Tuzson, L. Emmenegger, Y.-C. Chang, L. Hvozdara, H. P. Herzig, P. Wägli, A. Homsy,
N. F. de Rooij, A. Wirthmueller, D. Hofstetter, H. Looser and J. Faist, Analyst, 2014, 139, 2039-2046.

3 M. R. Ras, F. Borrull and R. M. Marcé, TrAC, Trends Anal. Chem., 2009, 28, 347-361.

4 G. Konvalina and H. Haick, Acc. Chem. Res., 2014, 47, 66-76.

5 D. Perez-Guaita, V. Kokoric, A. Wilk, S. Garrigues and B. Mizaikoff, J. Breath Res., 2014, 8, 026003.

6 T. Karl, P. Prazeller, D. Mayr, A. Jordan, J. Rieder, R. Fall and W. Lindinger, J. Appl. Physiol., 2001, 91, 762-770.

7 B. G. Stone, T. J. Besse, W. C. Duane, C. D. Evans and E. G. DeMaster, Lipids, 1993, 28, 705-708.

8 A. Cailleux and P. Allain, Life Sci., 1989, 44, 1877-1880.

9 T. P. Korman, B. Sahachartsiri, D. Li, J. M. Vinokur, D. Eisenberg and J. U. Bowie, Protein Sci., 2014, 23, 576-585.

10 C. Hornuss, A. Zagler, M. E. Dolch, D. Wiepcke, S. Praun, A.-L. Boulesteix, F. Weis, C. C. Apfel and G. Schelling, J. Breath Res., 2012, 6, 046004.

11 A. Wilk, J. C. Carter, M. Chrisp, A. M. Manuel, P. Mirkarimi, J. B. Alameda and B. Mizaikoff, Anal. Chem., 2013, 85, 1120511210.

12 J. C. Carter, M. P. Chrisp, A. M. Manuel, B. Mizaikoff, A. Wilk and S.-S. Kim, Substrate-integrated hollow waveguide sensors, U.S. Patent Application No. 13/631,936, September 29, (71), 2012.

13 J. da Silveira Petruci, P. R. Fortes, V. Kokoric, A. Wilk, I. M. Raimundo, A. A. Cardoso and B. Mizaikoff, Analyst, 2014, 139, 198-203.

14 A. Wilk, F. Seichter, S.-S. Kim, E. Tütüncü, B. Mizaikoff, J. A. Vogt, U. Wachter and P. Radermacher, Anal. Bioanal. Chem., 2012, 402, 397-404.

15 C. R. Young, N. Menegazzo, A. E. Riley, C. H. Brons, F. P. DiSanzo, J. L. Givens, J. L. Martin, M. M. Disko and B. Mizaikoff, Anal. Chem., 2011, 83, 6141-6147.

16 J. D. Fenske and S. E. Paulson, J. Air Waste Manage. Assoc., 1999, 49, 594-598.

17 S.-I. Ohira, J. Li, W. a. Lonneman, P. K. Dasgupta and K. Toda, Anal. Chem., 2007, 79, 2641-2649.

18 C. Grote and J. Pawliszyn, Anal. Chem., 1997, 69, 587-596.

19 B. Buszewski, J. Rudnicka, T. Ligor, M. Walczak, T. Jezierski and A. Amann, TrAC, Trends Anal. Chem., 2012, 38, 1-12.

20 J. Dummer, M. Storer, M. Swanney, M. McEwan, A. ScottThomas, S. Bhandari, S. Chambers, R. Dweik and M. Epton, TrAC, Trends Anal. Chem., 2011, 30, 960-967.

21 P. Eilers and H. Boelens, Leiden, Baseline Correction with Asymmetric Least Squares Smoothing, 2005, http:// zanran_storage.s3.amazonaws.com/www.science.uva.nl/ ContentPages/443199618.pdf.

22 F. Kühnemann, M. Wolfertz, S. Arnold, M. Lagemann, A. Popp, G. Schüler, A. Jux and W. Boland, Appl. Phys. B: Lasers Opt., 2002, 75, 397-403.

23 M. Traetteberg, G. Paulen, S. J. Cyvin, Y. N. Panchenko and V. I. Mochalov, J. Mol. Struct., 1984, 116, 141-151.

24 G. L. Long and J. D. Winefordner, Anal. Chem., 1983, 55, 712A-724A. 